\title{
Real-Time Multirate Filtering of Digitised Torque Signals on Tiva Mi- crocontroller using Fixed-Point Design with MATLAB
}

Jack A. Wilkie, Institute of Technical Medicine, Hochschule Furtwangen, Villingen-Schwenningen, Germany, wij@hsfurtwangen.de

Thomas Stieglitz, Department of Microsystems Engineering, University of Freiburg, Freiburg, Germany, stieg$\underline{\text { litz@imtek.uni-freiburg.de }}$

Knut Moeller, Institute of Technical Medicine, Hochschule Furtwangen, Villingen-Schwenningen, Germany, moe@hsfurtwangen.de

\section{Introduction}

Bone screws are used in various orthopaedic procedures to stabilise fractures and fix implants; correct screw torquing is critical to ensure resilience and longevity. To test models of bone screw insertion, a test rig was developed to collect data. The test rig requireds a torque sensor to collect accurate torque information to correlate with rotation and insertion depth. This sensor outputs an analog voltage, and is digitised by the 12-bit ADC on a TI TM4C123GH6PM. To reduce noise on the digitised signal and increase resolution through oversampling, a digital filter was used.

\section{Methods}

Due to the slow clock speed and simplicity of the microcontroller, care had to be taken to reduce the filters computational load. The $4 \mathrm{x}$ hardware-averaging of the microcontroller the first step of the filtering, reducing the data rate from $1 \mathrm{MHz}$ to $250 \mathrm{kHz}$, lowering the real-time computational requirements for the next step. Then an equiripple FIR filter was designed in MATLAB to filter the $250 \mathrm{kHz}$ signal with a passband frequency of $100 \mathrm{~Hz}$, a stopband frequency of $1 \mathrm{kHz}$, and stopband attenuation of $70 \mathrm{~dB}$; this allowed the signal to be subsequently decimated to $1 \mathrm{kHz}$ with negligible aliasing. This filter was quantized and implemented on the microcontroller using 627 16-bit fixed-point coefficients, and a scaling factor. DMA was used to copy ADC data efficiently to memory, and a FreeRTOS task processed the filter 1000 times per second.

\section{Results}

The implemented filter attenuated any signal above $1 \mathrm{kHz}$ by at least $60 \mathrm{~dB}$. Artefacts occur as spikes at 250 and 500 $\mathrm{kHz}$ due to hardware averaging. In practice this filtering methodology reduced the noise floor of the signal and increased the effective resolution of the ADC.

\section{Conclusion}

The designed digital filter worked effectively to filter noise and down-sample the signal efficiently in real-time. 


\section{Nonlinearity of Magnetostrictive Torque Sensor under Varying Exter- nal Magnetic Field Strength}

Jack Wilkie, Institute of Technical Medicine, Hochschule Furtwangen, Villingen-Schwenningen, Germany, wij@hsfurtwangen.de

Knut Moeller, Institute of Technical Medicine, Hochschule Furtwangen, Villingen-Schwenningen, Germany, moe@hsfurtwangen.de

\section{Introduction}

In order to test models of bone screw insertion, a test rig had to be developed to collect data. An integral part of this was the accurate recording of torque signals, and correlating these signals with screw rotation and insertion depth. The torque sensor we used was an NCTE 2300-20-1-AU-0-0; this sensor uses inverse-magnetorstrictive technology to sense torque ina rotating shaft. During use, we noted that external magnetic fields from screw holders or motors could be coupled into the sensor through connecting shafts. These fields caused a indisputable offset in the sensor reading. What was not so clear was the potential effect on the sensor linearity, which is addressed here.

\section{Methods}

To test the effects of of the magnetic field, we compared the output of the sensor with a different sensor based on a different technology unaffected by magnetic fields. The magnetic field strength was quantified with the zero-torque offset of the sensor, and was altered by adjusting the coupling spacing between the sensor shaft and magnetic screw-bit holder. In each configuration, the torque was ramped up and down 3-4 times to determine the relationship between the sensors

\section{Results}

Assuming the reference sensor was linear, we found that the configurations with higher magnetic field resulted in slightly greater non-linearities in the mangetostrictive sensor.

\section{Conclusion}

We found some evidence of non-linearity in our magnetostrictive torque sensor when exposed to external magnetic fields. However, the differences were small, so further research would be required to rule out other factors. 